\title{
Impact of Time Factors on Outcome in Patients with Head and Neck Cancer Treated with Definitive Radio(Chemo)Therapy
}

\author{
SÖREN DAHLKE ${ }^{1}$, DIANA STEINMANN ${ }^{1}$, HANS CHRISTIANSEN ${ }^{1}$, MARTIN DURISIN $^{2}$, \\ ANDRE ECKARDT $^{3}$, GERD WEGENER $^{4}$, MICHAEL BREMER ${ }^{1}$ and ANDREAS MEYER ${ }^{1}$ \\ Departments of ${ }^{1}$ Radiation Oncology, ${ }^{2}$ Otolaryngology and ${ }^{3}$ Cranio-Maxillo-Facial Surgery, and \\ ${ }^{4}$ Tumour Centre Hannover, Medical School Hannover, Hannover, Germany
}

\begin{abstract}
Aim: To evaluate treatment-related factors such as overall treatment time $(O T T)$ and radiation treatment time (RTT) in head-and-neck cancer. Patients and Methods: A total of 216 patients with locoregionally advanced inoperable head and neck cancer were treated with definitive radio(chemo)therapy. Mean follow-up was 37 months. Results: Median time from diagnosis to start of radiotherapy (total waiting time) was 34 days, and comprised of referral waiting time and time for preparatory work. Median RTT was 40 days, and median OTT was 91 days. At 6, 12 and 24 months local recurrence-free survival (LRFS) was 75\%, 65\% and 60\%; metastasis-free survival (MFS) was 84\%, $77 \%$ and $70 \%$; overall survival (OS) was $72 \%, 58 \%$ and $40 \%$. Tumor stage, boost and chemotherapy were significant for OS, waiting time for preparatory work and RTT were significant for MFS, and referral waiting time and total radiotherapy dose for LRFS. Conclusion: RTT $\leq 40$ days was a prognostic factor for better MFS. Prolonged waiting time had a converse effect for radiotherapy with better outcome on MFS and LRFS.
\end{abstract}

Squamous cell carcinoma of the head and neck (SCCHN) is a cancer with worldwide impact. Oral cavity cancer as subgroup of SCCHN for instance accounts for approximately 300,400 new cases and 145,400 deaths every year (1). Most of these cases, approximately $90 \%$, are SCCs. The most important risk factors in that etiology are tobacco and alcohol consumption, followed by other risk

This article is freely accessible online.

Correspondence to: Sören Dahlke, Department of Radiation Oncology, Medical School Hannover, Carl-Neuberg-Str. 1, 30625 Hannover, Germany. Tel: +49 5115322575, Fax: +49 5115329797, e-mail: Dahlke.Soeren@gmx.de

Key Words: Head and neck cancer, radio(chemo)therapy, localy advanced, inoperable. factors such as human papilloma virus infection, dietary factors and genetic susceptibility (2). Small SCCHN without lymphatic involvement can be cured in $70-90 \%$ of cases with either surgery or radiotherapy alone (3). For locally advanced SCCHN i.e. stage III or IV, surgery, radiotherapy and chemotherapy are the main treatment modalities for curative management. However, local and distant failure rates remain high despite intensified and combination therapy (4). If surgery is not possible due to co-morbidities or is refused due to potential loss of organ function, definitive radiotherapy with or without chemotherapy or targeted therapy e.g. cetuximab can be applied as a curative approach (5). The combination of therapies has shown to be superior in comparison to radiotherapy alone in several randomized studies and meta-analysis regarding overall survival and local control $(4,6,7)$.

Up to $40 \%$ of patients treated for head and neck cancer develop a recurrence within 5 years after treatment. Reirradiation should definitely be considered for patients with a recurrent or second primary head and neck cancer (8). Therefore, improved local control is likely to lead to improved disease-free and overall survival (OS) (9). There are different prognostic treatment factors with impact on the further course of disease such as simultaneous application of chemotherapy or targeted therapy and total dose, dose per fraction or fractions per day/per week $(10,11)$. As well as these established factors, there may be other treatment-related factors with influence on further outcome, such as treatment time. In other cancer types, a prolonged overall treatment time (OTT) had a negative impact on survival (12). The influence of time as a factor may also become more important in countries with an imbalance between increased demand and availability of radiotherapy treatment units (13). Therefore, in addition to established prognostic factors, we analyzed the influence of different time periods influencing the OTT on further outcome of disease in patients with locally advanced SCCHN in terms of locoregional recurrence-free survival (LRFS), metastasis-free survival (MFS) and OS. 


\section{Patients and Methods}

From $10 / 1996$ to $09 / 2009,216$ patients (female $n=180$, male $n=36$ ) with locally advanced SCCHN were treated at our Institution with definitive treatment with and without chemotherapy and were included in this analysis. Clinical data were obtained retrospectively by evaluation of all patients' records, including the radiation oncologist's records on follow-up visits. Furthermore, additional data were obtained from the Regional Tumor Center Hannover. All primary tumors were histologically proven by biopsy. Local and regional extension of the tumor was staged according to the fourth, fifth and sixth editions of TNM (14-16). All patients underwent computed tomography-based treatment planning after immobilization by mask fixation with 3D conformal therapy. The target volume included all gross disease plus a safety margin to account for setup uncertainties. The median total dose of irradiation treatment was 66.6 Gy (range $=59.4-72.4$ Gy). The patients' characteristics, time intervals and further details are summarized in Table I.

Total waiting time was defined as the interval from the date of biopsy to the start of radiotherapy according to León et al. (17). For further analysis, the waiting time was split into two further intervals: referral waiting time, defined as the interval from biopsy to date of the first visit to the Radiation Department; and the waiting time for preparatory work, defined as the interval between the first visit to the Radiation Department and start of radiotherapy. Radiation treatment time (RTT) was defined as the time interval from the first to the last fraction of the radiotherapy, and OTT is defined as the time interval from the date of biopsy until the end of irradiation.

Statistical analysis was performed using a commercially available software package (SPSS 21.0 for Windows; IBM Corp., Armonk, NY, USA). All events were measured from the end of irradiation treatment. The following end-points were analysed: LRFS, MFS and OS. The endpoints were compared between following subgroups: gender (male versus female), age ( $>58$ versus $\leq 58$ years), total dose of irradiation ( $>66.6$ versus $\leq 66.6 \mathrm{~Gy}$ ), simultaneous application of chemotherapy (yes versus no), tumor stage (T1/2 versus $\mathrm{T} 3 / 4)$, lymph node status (N0 versus $\mathrm{N} 1 / 2 / 3$ ), concomitant boost (yes versus no), neck dissection (yes versus no), total waiting time ( $>34$ versus $\leq 34$ days), referral waiting time ( $>16$ versus $\leq 16$ days), waiting time for preparatory work ( $>14$ versus $\leq 14$ days), RTT ( $>40$ versus $\leq 40$ days), OTT (>91 versus $\leq 91$ days). The actuarial rates were calculated by the product-limit method of Kaplan and Meier, differences were compared using the log-rank test. Differences with a $p$-value of less than 0.05 were considered statistically significant. A multivariate step-wise Cox proportional regression analysis was used to identify significant prognostic factors for the clinical endpoints analyzed. The following parameters were included in the analysis: gender, age at time of irradiation, tumor stage, lymph node status, concomitant radiotherapy boost, use of simultaneous chemotherapy, neck dissection, referral waiting time, waiting time for preparatory work, RTT and total radiotherapy dose.

\section{Results}

Of the 216 patients treated with radiotherapy, 180 were male $(83.3 \%)$ and 36 female $(16.7 \%)$. The median age of the patients at the time of irradiation was 58 years (range $=36-84$ years). Overall, 93 patients were treated with definite radiotherapy alone (43\%), and 123 patients with radiotherapy
Table I. Patient characteristics including time intervals.

\begin{tabular}{|c|c|}
\hline Characteristic & Value \\
\hline \multicolumn{2}{|l|}{ Age, years } \\
\hline Median (range) & $58(36-84)$ \\
\hline \multicolumn{2}{|l|}{ Gender, n (\%) } \\
\hline Male & $180(83.3 \%)$ \\
\hline Female & $36(16.7 \%)$ \\
\hline \multicolumn{2}{|l|}{ T-Stage, $\mathrm{n}(\%)$} \\
\hline $\mathrm{T} 1$ & $2(0.9 \%)$ \\
\hline $\mathrm{T} 2$ & $16(7.4 \%)$ \\
\hline T3 & $52(24.1 \%)$ \\
\hline $\mathrm{T} 4$ & $146(67.6 \%)$ \\
\hline \multicolumn{2}{|l|}{ N-Stage, n (\%) } \\
\hline No & $35(16.2 \%)$ \\
\hline $\mathrm{N} 1$ & $51(23.6 \%)$ \\
\hline $\mathrm{N} 2$ & $119(55.1 \%)$ \\
\hline N3 & $11(5.1 \%)$ \\
\hline \multicolumn{2}{|l|}{ Tumor site, $\mathrm{n}(\%)$} \\
\hline Oropharynx/oral cavity & $78(36.1 \%)$ \\
\hline Hypopharynx & $114(52.8 \%)$ \\
\hline Larynx & $24(11.1 \%)$ \\
\hline \multicolumn{2}{|l|}{ Concomitant -boost, n (\%) } \\
\hline Yes & $42(19.4 \%)$ \\
\hline No & $174(80.6 \%)$ \\
\hline \multicolumn{2}{|l|}{ Chemotherapy, n (\%) } \\
\hline Yes & $123(56.9 \%)$ \\
\hline No & $93(43.1 \%)$ \\
\hline \multicolumn{2}{|l|}{ Neck dissection before RT, n (\%) } \\
\hline Yes & $43(19.9 \%)$ \\
\hline No & $173(80.1 \%)$ \\
\hline \multicolumn{2}{|l|}{ Median time interval (range), days } \\
\hline Referral waiting time & $16(0-70)$ \\
\hline Waiting for preparatory work & $14(2-76)$ \\
\hline Total waiting time & $34(11-89)$ \\
\hline RTT & $40(29-66)$ \\
\hline OTT & $91(62-139)$ \\
\hline
\end{tabular}

RTT: Radiation treatment time; OTT: overall treatment time.

and simultaneous application of chemotherapy (57\%). In 35 patients $(16 \%)$ an accelerated fractionation scheme with concomitant boost was carried out. Simultaneous application of chemotherapy consisted of cisplatin alone in 74 patients (34.2\%), cisplatin/5-fluorouracil in $23(10.6 \%)$, gemcitabine in $20(9.3 \%)$, carboplatin/taxol in four $(1.8 \%)$ and carboplatin alone in two $(0.9 \%)$. Targeted therapy, such as cetuximab, was not used in this cohort of patients. The median total dose of irradiation was 66.6 Gy (range=59.4-72.4 Gy). The different time intervals are detailed in Table I.

Median follow-up time was 37 months (range=0-166 months). The short follow up of 0 months was due to the fact that seven patients died shortly after or during treatment due to advanced tumor $(n=4)$, poor general condition $(n=1)$, fulminant pulmonary embolism $(n=1)$ or hemorrhage $(n=1)$. During follow-up, 75 (34.7\%) of patients developed 
Table II. Univariate and the multivariate regression analyses of the various factors associated with 6- and 24-month data for overall l (OS), metastasis-free (MFS) and locoregional recurrence-free (LRFS) survival of patients.

\begin{tabular}{|c|c|c|c|c|c|c|c|c|c|c|c|c|}
\hline \multirow[b]{2}{*}{ Factor } & \multicolumn{2}{|c|}{ OS $(\%)$} & \multicolumn{2}{|c|}{$p$-Value } & \multicolumn{2}{|c|}{ MFS (\%) } & \multicolumn{2}{|c|}{$p$-Value } & \multicolumn{2}{|c|}{ LRFS (\%) } & \multicolumn{2}{|c|}{$p$-Value } \\
\hline & $\begin{array}{c}\text { 6- } \\
\text { Month }\end{array}$ & $\begin{array}{l}\text { 24- } \\
\text { Month }\end{array}$ & Univariate & Multivariate & $\begin{array}{c}\text { 6- } \\
\text { Month }\end{array}$ & $\begin{array}{c}24- \\
\text { Month }\end{array}$ & Univariate & Multivariate & $\begin{array}{c}\text { 6- } \\
\text { Month }\end{array}$ & $\begin{array}{c}24- \\
\text { Month }\end{array}$ & Univariate & Multivariate \\
\hline Overall & & 72 & 40 & & & 84 & 70 & & & 75 & 60 & \\
\hline \multicolumn{13}{|l|}{ Gender } \\
\hline Male & 72 & 38 & 0.242 & 0.524 & 83 & 68 & 0.097 & 0.169 & 75 & 56 & 0.071 & 0.120 \\
\hline Female & 75 & 49 & & & 93 & 81 & & & 76 & 76 & & \\
\hline \multicolumn{13}{|l|}{ Age } \\
\hline$\leq 58$ Years & 74 & 44 & 0.018 & 0.177 & 87 & 69 & 0.547 & 0.969 & 73 & 59 & 0.752 & 0.649 \\
\hline$>58$ Years & 70 & 35 & & & 80 & 71 & & & 77 & 60 & & \\
\hline \multicolumn{13}{|l|}{ Tumor stage } \\
\hline $\mathrm{T} 1 / 2$ & 70 & 60 & 0.018 & 0.010 & 89 & 89 & 0.112 & 0.099 & 84 & 76 & 0.209 & 0.102 \\
\hline $\mathrm{T} 3 / 4$ & 72 & 37 & & & 84 & 67 & & & 74 & 58 & & \\
\hline \multicolumn{13}{|l|}{ Nodal stage } \\
\hline No & 74 & 49 & 0.593 & 0.280 & 91 & 83 & 0.133 & 0.101 & 80 & 57 & 0.980 & 0.576 \\
\hline $\mathrm{N}+$ & 72 & 38 & & & 83 & 67 & & & 74 & 60 & & \\
\hline \multicolumn{13}{|c|}{ Concomitant RT boost } \\
\hline Yes & 90 & 67 & 0.000 & 0.003 & 90 & 82 & 0.257 & 0.914 & 77 & 74 & 0.264 & 0.484 \\
\hline No & 68 & 33 & & & 82 & 65 & & & 75 & 54 & & \\
\hline \multicolumn{13}{|c|}{ Chemotherapy } \\
\hline Yes & 82 & 50 & 0.000 & 0.004 & 85 & 70 & 0.992 & 0.698 & 80 & 68 & 0.047 & 0.257 \\
\hline No & 59 & 26 & & & 83 & 69 & & & 66 & 43 & & \\
\hline \multicolumn{13}{|c|}{ ND before RT } \\
\hline Yes & 72 & 56 & 0.144 & 0.574 & 86 & 82 & 0.244 & 0.824 & 75 & 65 & 0.847 & 0.506 \\
\hline No & 72 & 35 & & & 84 & 66 & & & 75 & 58 & & \\
\hline \multicolumn{13}{|l|}{$\mathrm{RWT}^{*}$} \\
\hline$\leq 16$ Days & 71 & 32 & 0.011 & 0.098 & 89 & 67 & 0.641 & 0.416 & 68 & 50 & 0.010 & 0.032 \\
\hline >16 Days & 73 & 48 & & & 89 & 71 & & & 83 & 70 & & \\
\hline \multicolumn{13}{|l|}{ WPW* } \\
\hline$\leq 14$ Days & 72 & 35 & 0.444 & 0.537 & 79 & 63 & 0.004 & 0.016 & 74 & 53 & 0.242 & 0.316 \\
\hline >14 Days & 72 & 44 & & & 89 & 76 & & & 76 & 65 & & \\
\hline \multicolumn{13}{|l|}{ RTT* } \\
\hline$\leq 40$ Days & 80 & 47 & 0.013 & 0.723 & 91 & 81 & 0.001 & 0.007 & 77 & 69 & 0.060 & 0.527 \\
\hline >40 Days & 63 & 30 & & & 72 & 50 & & & 73 & 44 & & \\
\hline \multicolumn{13}{|l|}{ Total dose* } \\
\hline$\leq 66.6 \mathrm{~Gy}$ & 66 & 31 & 0.004 & 0.618 & 83 & 64 & 0.307 & 0.370 & 69 & 48 & 0.015 & 0.048 \\
\hline$>66.6 \mathrm{~Gy}$ & 80 & 51 & & & 85 & 75 & & & 82 & 72 & & \\
\hline
\end{tabular}

ND: Neck dissection; RT: radiotherapy; RWT: referral waiting time; WPW: waiting for preparatory work; RTT: radiation treatment time.*Cut-offs set at median value.

locoregional recurrent tumor after a median of 7 months and $48(22.2 \%)$ patients developed distant metastasis after a median of 7 months. Overall $170(78.7 \%)$ patients died during the follow-up period. LRFS after 6, 12 and 24 months was $75 \%, 65 \%$ and $60 \%$, MFS was $84 \%, 77 \%$ and $70 \%$, and OS was $72 \%, 58 \%$ and $40 \%$, respectively. Further survival rates according to subgroups are given in Table II.

Univariate analysis of OS showed the following factors to be statistically significant: age $(p=0.018)$, stage of tumor $(p=0.018)$, concomitant boost $(p<0.001)$, chemotherapy $(p<0.001)$, referral waiting time $(p=0.011)$, RTT $(p=0.013$, Figure 1) and total radiotherapy dose $(p=0.004)$. In multivariate analysis, the following factors remained statistically significant: tumor stage $(p=0.010)$, boost $(p=0.003)$, and simultaneous chemotherapy $(p=0.004)$. The 2 -year OS was $60 \%$ versus $37 \%$ for those with tumor stage $\mathrm{T} 1 / 2$ versus those with $\mathrm{T} 3 / 4,67 \%$ versus $33 \%$ for those with versus without concomitant boost, and $50 \%$ versus $26 \%$ for those treated with simultaneous chemotherapy versus those treated with irradiation alone.

For MFS, waiting time for preparatory work $(p=0.004)$ and RTT ( $p=0.001)$ were statistically significant in univariate and multivariate analyses. The 2-year metastasis-free survival was $63 \%$ versus $76 \%$ for waiting time for preparatory work of $\leq 14$ versus $>14$ days and $81 \%$ versus $50 \%$ for RTT of $\leq 40$ versus $>40$ days. 


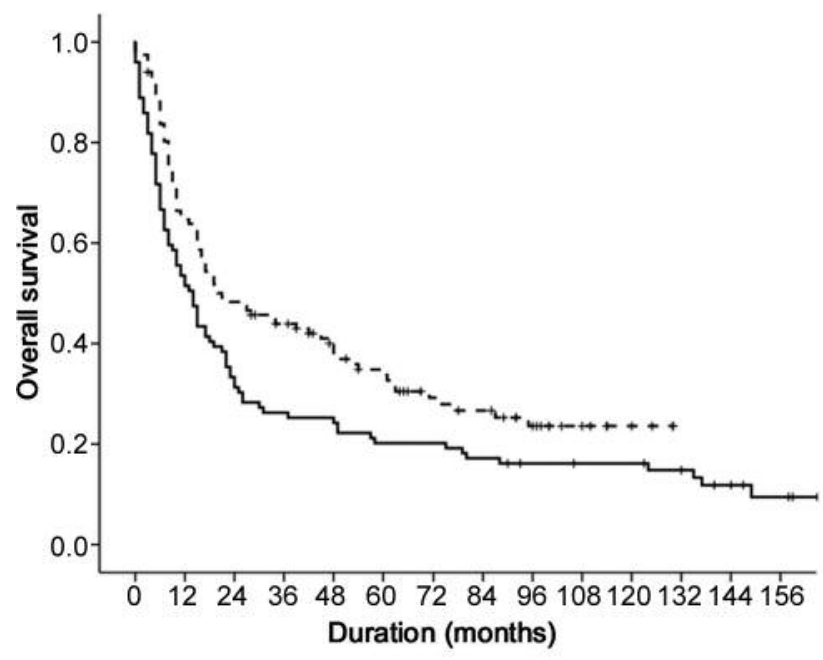

Figure 1. Overall survival of patients with $\leq 40$ days (broken line, $n=117$ ) versus those with $>40$ days (solid line, $n=99$ ) of radiation treatment time (RTT), calculated by Kaplan-Meier method (log-rank test, $p=0.013)$.

Radiotherapy total dose $(p=0.015)$ was statistically significant in univariate analysis. In multivariate analysis, referral waiting time $(p=0.032)$ and total radiotherapy dose $(p=0.048)$ remained statistically significant, with a 2 -year LRFS of $50 \%$ versus $70 \%$ for referral waiting time of $\leq 16$ versus $>16$ days and $48 \%$ versus $72 \%$ for total radiotherapy dose $\leq 66.6$ versus $>66.6$ Gy.

\section{Discussion}

In the treatment of patients with cancer, different prognostic factors with impact on outcome of therapy and further course of disease can be found (18). Prognostic factors can be divided into three different groups: tumor-related, such as tumor stage; patient-related, such as gender or age; and treatment-related prognostic factors such as treating physician, healthcare system and treatment delivery $(11,18$, 19). All different factors may have an impact and can interact with each other, allowing a prediction of outcome for every single patient, individually.

Well-known and established factors are tumor stage as a tumor-related factor, gender as a patient-related factor and total radiation dose and simultaneous application of chemotherapy as treatment-related factors $(2,6,7,20)$. We clearly demonstrated an impact of simultaneous application of chemotherapy on OS, with a 2 -year OS of $50 \%$ versus $26 \%$ for radiotherapy alone that is also in the range reported by other authors $(6,21)$.

However, there may be other treatment-related factors with an impact on patient's outcome such as treatment time intervals. This becomes more interesting in countries with an imbalance between demand and availability of radiotherapy treatment units. Different authors have evaluated the total waiting time defined as the time interval from initial diagnosis to start of irradiation and found different results.

Due to the locally aggressive behavior of head and neck cancer cells, the association between increased waiting time and increased risk of cancer growth may result in a higher tumor stage, leading to worse outcome. This was shown by Jensen et al. in 414 patients treated with primary radiotherapy from 2000-2005. Within a waiting time interval of 2-4 weeks, $68 \%$ of patients showed measurable tumor progression, and after 4 weeks, $70 \%$ of patients had an increase in total tumor volume (22). A mean increase in tumor volume of $70 \%$ during a mean waiting time of 56 days was also found by Waajier et al. (23).

A reduction of the tumor control rate of $8 \%$ with a tumor volume doubling time is also described by Mackillop et al. for a treatment delay of 4 weeks (24).

Regarding the waiting time for definitive treatment, Lyhne et al. compared waiting times between diagnosis and treatment of head and neck cancer in different time periods: 2010 (253 patients), 2002 (211 patients) and 1992 (168 patients) (25). The waiting time was further divided into pre-diagnostic time (time to diagnosis or referral waiting time) and post-diagnostic time (time from diagnosis to treatment or waiting for preparatory work. The authors found a total waiting time of 25 days in 2010 versus 47 days in $2002(p<0.001)$ and 31 days in $1992(p<0.001)$. This significant reduction in delay of treatment of head and neck cancer in 2010 arose after a Danish national policy of fast track accelerated clinical pathways was implemented. However, this reduction in waiting time in 2010 has not been compared for outcomes of patients. Still, the influence of the healthcare system and available health resources as well as a possible impact on OTTs was demonstrated. Fortin et al. divided his cohort of 623 patients into three different waiting time intervals: $<30$ days, 31-40 days, and $>40$ days. The 3-year locoregional control rate was $80 \%, 75 \%$, and $40 \%$, and the neck-disease control was $91 \%, 89 \%$, and $79 \%$, respectively. They described that a waiting time of over 40 days was correlated with significant increased risk of local and neck failure, as well as a decrease of OS rate of $15 \%$ compared with other patients (26). Chen et al. conducted a systematic review of the literature between 1975 and 2005 for the effect of waiting time of radiotherapy for different cancers on clinical outcomes (27). They identified 44 relevant studies $(26,231$ patients) and demonstrated in their meta-analyses of 20 studies (12,463 patients) a significant increase in the risk of local failure with increasing waiting time. Of these 20 studies, 13 examined patients with head and neck cancer in the period 1990-2004. Chen et al. demonstrated an absolute increase in the risk of recurrence for of $3.7 \%$ per month of delay of definitive radiotherapy. 
This resulted in a marginally significant decrease in survival with a relative risk for death/month of 1.16 . The authors concluded that the risk of local recurrence increases with increasing waiting time, and that the increase in local recurrence rates may translate into reduced survival in some clinical situations.

A more recent investigation by van Harten et al. with 13,140 patients described a median waiting time of 37 days. In this study, patients from the Netherlands were treated with surgery, radiotherapy and chemoradiation. The 5-year OS was $58 \%$ for patients overall. Multivariable Cox regression showed that longer waiting time ( $>30$ days) was related to a higher hazard of dying $(p<0.0001)$. They concluded that a longer waiting time is a significant negative prognostic factor (28).

Another current study by Stoker et al. with 142 patients (only nasopharyngeal cancer) demonstrated a median waiting time of 117 days. The 2-year local regional control and disease-free survival were $48 \%$ and $32 \%$. Univariate Cox analysis showed a significant hazard for poor local regional control and disease-free survival when the waiting time increased to $>130$ days. There was no association between long waiting time and OS (29).

Other authors did not detect any influence of the waiting time on further outcome of disease. In a study conducted by Lee et al. examining 209 patients with nasopharyngeal carcinoma, the patients were classified into three groups with respect to the delay caused by waiting: $<22$ days, $22-28$ days and $>28$ days. The 10-year LRFS rates for the three groups were $76 \%, 80 \%$, and $82 \%$, respectively, without any statistically significant difference (30). Brouha et al. defined a waiting time interval as being from positive histopathological diagnosis to the start of radiotherapy (waiting time for preparatory work) of 43 days for 362 patients with glottic laryngeal carcinoma in the Netherlands during 1980-1996, with an actuarial recurrence-free rate of $82.5 \%$ at 5 years. They also did not demonstrate any effect of the waiting time on outcome (13). Leon et al. examined the influence of waiting time for radiotherapy of 797 patients with SCCHN treated between 1985 and 1998. They found a median waiting time period from biopsy to start of radiotherapy (total waiting time) of 44 days. They divided this waiting time into a period from biopsy to first visit to the Radio-oncology Department (referral waiting time) with a median of 14 days and from first visit to start of radiotherapy (waiting time for preparatory work) with a median of 28 days. The actuarial 5-year LRFS was $73 \%$, the regional recurrence-free survival was $92 \%$ and the diseasespecific survival was $85 \%$. There were no significant differences either in the locoregional failure or diseasespecific survival in relation to the waiting time (17).

We found a median total waiting time of 34 days (range $=11-89$ days), which is in line with the findings above. Further dividing the waiting time into a referral waiting time and waiting time for preparatory work gave a median of 16 and 14 days, respectively. However, we detected a statistically significant influence both in uni- and multivariate analysis for referral waiting time on LRFS and for waiting time for preparatory work on metastasis-free survival, with an inverse influence. The 2-year LRFS was $50 \%$ versus $70 \%$ for $\leq 16$ versus $>16$ days referral waiting time and $76 \%$ versus $63 \%$ for $\leq 14$ versus $>14$ days waiting time for preparatory work. OS was not influenced statistically significantly by any of the time periods in multivariate analysis. The inverse influence of prolonged waiting times for radiotherapy and better outcome has been seen for other tumor entities. Crawford et al. evaluated the outcome of patients with endometrial cancer and found that patients who experienced the longest delay in treatment were more likely to survive (31). In 2,855 patients with glioblastoma multiforme, Blumenthal et al. found statistically significant longer survival in patients with the longest interval ( $>4$ weeks) between surgery and the beginning of radiotherapy compared to those with the shortest delay ( $\leq 2$ weeks) (32). However, it may be possible that larger and more aggressive tumors may have received priority in treatment, resulting in shorter waiting times on the one hand. On the other hand, it may also be that patients with a poor performance status are irradiated with simple techniques in a rapid way, whereas patients with good performance status are prepared for more complex irradiation techniques that necessitate more preparation time.

Regarding the RTT, i.e. radiotherapy without long breaks or gaps, Sher et al. analyzed an RTT $\leq 51$ days versus $>51$ days on OS for locally advanced SCCHN. They found a prolonged RTT $>51$ days to be associated with an inferior overall survival (hazard ratio $=1.63, p=0.0058$ ). For each additional day required to finish radiotherapy beyond 8 weeks, the hazard rate of death increased by $4.2 \%$ (33). Barton et al. evaluated 581 patients with early laryngeal cancer and found that a longer RTT was statistically significantly associated with reduced local control in multivariate analysis. Their treatment durations ranged from 16 to 117 days, with a median of 46 days (20).

Cannon et al. reviewed outcomes for 171 patients with head and neck cancer treated with curative radiotherapy and concurrent drug therapy. The median RTT was 46 days. Patients with RTT $\leq 49$ days had a superior 3-year local control rate and OS compared to those with RTT $>49$ days (88\% versus 71 and $81 \%$ versus $58 \%$, respectively) (34).

Stoker et al. found an RTT of 58 days. RTT was not associated with any of the clinical outcome parameters (29).

However, delayed waiting time and RTT would be expected to have an effect on the outcome of patients with SCCHN. These two time periods can be summarized as the OTT. We also investigated the impact of the OTT on outcome. We found a median OTT of 91 days (range $=62-139$ days). This is 
clearly lower than the 21.3 weeks found by Sher $e t$ al. We did not detect any statistically significant difference for patients between longer and shorter OTTs (OTT $>91$ days versus $\leq 91$ days) regarding LRFS, MFS or OS. Sher et al. also analyzed the relationship between OTT and LRFS and OS. The cohort of 333 patients with locally advanced SCCHN was created by a secondary analysis of the TAX 324 trial. The median OTT was dichotomized but without any statistically significant difference in metastasis-free or OS (33).

In our investigation, we found a median RTT of 40 days to be a statistically significant factor in univariate analysis for OS, with a 2-year OS rate of $47 \%$ versus $30 \%$. Furthermore, the RTT was statistically significant in uni- and multivariate analyses for MFS, with a 2 -year rate of $81 \%$ versus $50 \%$. Therefore, we clearly demonstrate the necessity of radiotherapy without gaps or delays from first to last treatment for improved outcome and control of disease progression.

It should be noted that in our retrospective analysis, we compared different localizations and tumor stages of SCCHN on the one hand and different treatment schemes on the other hand, according to waiting times. This could be a reason for diverging waiting times. Our findings are in line with those of van Harten et al. who also demonstrated that patients who were treated in a large head and neck center (e.g. university setting) had a significantly lower hazard of dying, but had a longer waiting time (28).

To conclude, we were able to confirm that established prognostic factors such as simultaneous application of chemotherapy influence the outcome of radiotherapy for SCCHN. Additionally, a shorter RTT without gaps or breaks improves MFS. The impact of the waiting time for the start of the radiotherapy is very important. It may be that a more proper and longer preparation for radiotherapy may lead to a shorter RTT with better outcome.

\section{References}

1 Torre LA, Bray F, Siegel RL, Ferlay J, Lortet-Tieulent J and Jemal A: Global cancer statistics 2012. CA Cancer J Clin 65(2): 87-108, 2015.

2 Matzinger O, Zouhair A, Mirimanoff RO and Ozsahin M: Radiochemotherapy in locally advanced squamous cell carcinomas of the head and neck. Clin Oncol 21(7): 525-531, 2009.

3 Agrawal N and Ha PK: Management of early-stage laryngeal cancer. Otolaryngol Clin North Am 41(4): 757-769, 2008.

4 Ko C and Citrin D: Radiotherapy for the management of locally advanced squamous cell carcinoma of the head and neck. Oral Dis 15(2): 121-132, 2009.

5 Scher RL and Esclamado RM: Organ and function preservation: the role of surgery as the optimal primary modality or as salvage after chemoradiation failure. Semin Radiat Oncol 19(1): 17-23, 2009.

6 Pignon JP, Bourhis J, Domenge C and Designé L: Chemotherapy added to locoregional treatment for head and neck squamouscell carcinoma: three meta-analyses of updated individual data.
MACH-NC Collaborative Group. Meta-analysis of chemotherapy on head and neck cancer. Lancet 355(9208): 949$955,2000$.

7 Pignon JP, le Maître A and Bourhis J: Meta-Analyses of Chemotherapy in Head and Neck Cancer (MACH-NC): an update. Int J Radiat Oncol Biol Phys 69(2 Suppl): 112-114, 2007.

8 Bots WTC, van den Bosch S, Zwijnenburg EM, Dijkema T, van den Broek GB, Weijs WLJ, Verhoef LCG and Kaanders JHAM: Reirradiation of head and neck cancer: long-term disease control and toxicity. Head Neck 39(6): 1122-1130, 2017.

9 Wong LY, Wei WI, Lam LK and Yuen AP: Salvage of recurrent head and neck squamous cell carcinoma after primary curative surgery. Head Neck 25(11): 953-959, 2003.

10 Herrmann T and Baumann M: Prolongation of latency or overall treatment time by unplanned radiation pauses. The clinical importance of compensation. Strahlenther Onkol 181(2): 65-76, 2005.

11 Gospodarowicz M, Mackillop WJ, O'Sullivan B, Sobin L, Henson D, Hutter RV and Wittekind C: Prognostic factors in clinical decision making: The future. Cancer 91(8 Suppl): 18881895, 2001.

12 Richards MA, Westcombe AM, Love SB, Littlejohns P and Ramirez AJ: Influence of delay on survival in patients with breast cancer: a systematic review. Lancet 353(9159): 111911126, 1999.

13 Brouha XDR, Op De Coul B, Terhaard CJ and Hordijk GJ: Does waiting time for radiotherapy affect local control of T1NOM0 glottic laryngeal carcinoma? Clin Otolaryngol 25(3): 215-218, 2000.

14 Sobin LH and Wittekind C (eds): International Union Against Cancer (UICC): TNM Classification of Malignant Tumours. Sixth Edition. New York: Wiley, 2002.

15 Hermanek P and Sobin LH (eds): International Union Against Cancer (UICC): TNM Classification of Malignant Tumours. Fourth Edition. New York: 1987, revised 1992.

16 Sobin LH and Wittekind C (eds): International Union Against Cancer (UICC): TNM Classification of Malignant Tumours. Fifth Edition. New York: John Wiley \& Sons, Inc., 1997.

17 Leon X, de Vega M, Orús C, Morán J, Vergés J and Quer M: The effect of waiting time on local control and survival in head and neck carcinoma patients treated with radiotherapy. Radiother Oncol 66(3): 277-281, 2003.

18 Stockler M, Boyd N and Tannock I: Guide to studies of diagnostic tests, prognostic factors and treatments. In: The Basic Science of Oncology. Third Edition. Tannock I and Hill R (eds.). Toronto, MacGraw-Hill, pp. 466-492, 1998.

19 Gelb AB: Renal cell carcinoma: current prognostic factors. Union Internationale Contre le Cancer (UICC) and the American Joint Committee on Cancer (AJCC). Cancer 80(5): 981-986, 1997.

20 Barton MB, Morgan G, Smee R, Tiver KW, Hamilton C and Gebski V: Does waiting time affect the outcome of larynx cancer treated by radiotherapy? Radiother Oncol 44(2): 137144, 1997.

21 Blanchard P, Baujat B, Holostenco V, Bourredjem A, Baey C, Bourhis $\mathrm{J}$ and Pignon JP: MACH-CH Collaborative group. Meta-Analyses of Chemotherapy in Head and Neck Cancer (MACH-NC): A comprehensive analysis by tumour site. Radiother Oncol 100(1): 33-40, 2011. 
22 Jensen AR, Nellemann HM and Overgaard J: Tumor progression in waiting time for radiotherapy in head and neck cancer Radiother Oncol 84(1): 5-10, 2007.

23 Waaijer A, Terhaard CH, Dehnad H, Hordijk GJ, van Leeuwen MS, Raaymakers CP and Lagendijk JJ: Waiting times for radiotherapy: consequences of volume increase for the TCP in oropharyngeal carcinoma. Radiother Oncol 66(3): 271-276, 2003.

24 Mackillop WJ, Bates JH, O'Sullivan B and Withers HR: The effect of delay in treatment on local control by radiotherapy. Int J RadiatOncol Biol Phys 34(1): 243-250, 1996.

25 Lyhne NM, Christensen A, Alanin MC, Bruun MT, Jung TH, Bruhn MA, Jespersen JB, Kristensen CA, Andersen E, Godballe C, Buchwald C, Bundgaard T, Johansen J, Lambertsen K, Primdahl H, Toustrup K, Sørensen JA, Overgaard J and Grau C: Waiting times for diagnosis and treatment of head and neck cancer in Denmark in 2010 compared to 1992 and 2002. Eur J Cancer 49(7): 1627-1633, 2013.

26 Fortin A, Bairati I, Albert M, Moore L, Allard J and Couture C: Effect of treatment delay on outcome of patients with earlystage head-and-neck carcinoma receiving radical radiotherapy. Int $\mathrm{J}$ Radiat Oncol Biol Phys 52(4): 929-936, 2002.

27 Chen Z, King W, Pearcey R, Kerba M and Mackillop WJ: The relationship between waiting time for radiotherapy and clinical outcomes: A systematic review of the literature. Radiother Oncol 87(1): 3-16, 2008.

28 van Harten MC, Hoebers FJ, Kross KW, van Werkhoven ED, van den Brekel MW and van Dijk BA: Determinants of treatment waiting times for head and neck cancer in the Netherlands and their relation to survival. Oral Oncol 51(3): 272-278, 2015.

29 Stoker SD, Fles R, Herdini C, Rijntjes FJ, Tjokronagoro M, Dwidanarti SR, Sikorska K, Leemans CR, Schmidt MK, AlMamgani A, Wildeman MA, Haryana SM, Indrasari SR and Tan IB: The impact of the overall radiotherapy time on clinical outcome of patients with nasopharyngeal carcinoma; a retrospective study. PLoS One 11(3): 0151899, 2016.
30 Lee AWM, Chan DKK, Fowler JF, Poon YF, Law SC, Foo W, O SK, Tung SY, Cheung FK and Ho JH: T1 nasopharyngeal carcinoma: the effect of waiting time on tumor control. Int J Radiat Oncol Biol Phys 30(5): 1111-1117, 1994.

31 Crawford SC, Davis JA, Siddiqui NA, de Caestecker L, Gillis CR, Hole D and Penney G: The waiting time paradox: population based retrospective study of treatment delay and survival of women with endometrial cancer in Scotland. BMJ. 325(7357): 196, 2002.

32 Blumenthal DT, Won M, Mehta MP, Curran WJ, Souhami L, Michalski JM, Rogers CL and Corn BW: Short delay in initiation of radiotherapy may not affect outcome of patients with glioblastoma: A secondary analysis from the Radiation Therapy Oncology Group database. J Clin Oncol 27(5): 733-739, 2009.

33 Sher DJ, Posner MR, Tishler RB, Sarlis NJ, Haddad RI, Holupka EJ and Devlin PM: Relationship between radiation treatment time and overall survival after induction chemotherapy for locally advanced head-and-neck carcinoma: a subset analysis of TAX 324. Int J Radiat Oncol Biol Phys 81(5): 813-818, 2011.

34 Cannon DM, Geye HM, Hartig GK, Traynor AM, Hoang T, McCulloch TM, Wiederholt PA, Chappell RJ and Harari PM: Increased local failure risk with prolonged radiation treatment time in head and neck cancer treated with concurrent chemotherapy. Head Neck 36(8): 1120-1125, 2014.

Received June 9, 2017

Revised July 7, 2017

Accepted July 10, 2017 